\title{
Design of Seat Search System in the Classroom Based on Wireless Sensor Networks
}

\author{
https://doi.org/10.3991/ijoe.v14i01.7986 \\ Yuchen Jia( $\left({ }^{\bowtie}\right)$ \\ Agricultural University of Hebei, Baoding, China \\ jiayuchen1981@163.com \\ Mengxuan Cui \\ Beijing Institute of Technology, Beijing, China
}

\begin{abstract}
The purpose of this design is intended to statistics and publishes free seats information in classroom timely to students, and then save students' time to looking for classroom. The system uses wireless sensor networks to monitor classroom vacancies. It consists of classroom monitoring system and information transmission system. The classroom monitoring system consists of a coordinator node for remote wireless communication and two collection nodes for local communications in the classroom, and that three nodes are starconnected. The tasks of the coordinator node are to collect information from the collection nodes and display and transmission. Set up two collection nodes for collecting information of the number who inter the classroom. The devices for counting include two units, signal acquisition unit is constituted with pyroelectric infrared sensor which contains RE200B probe and conditioning circuit, and the control unit is constituted with CC2530 for signal processing. LCD screen is used to real-time display in coordinator node for counting the number of coming in or out the classroom. Users who enter the teaching building check which classroom have seats available. The manner of local communication is using ZIGBEE. The entire system uses sensor technology and mobile network communication technology to achieve real-time acquisition and release of information. The ability to identify and stability of the experimental system currently implemented are strong.
\end{abstract}

Keywords-Pyroelectric infrared sensor; Counting device; CC2530; ZIGBEE; WIFI

\section{$1 \quad$ Introduction}

In recent years, with the development of college education, the number of students increases a lot, teaching hardware resource utilization is higher than before, resulting in classroom resources are increasingly strained. Many students often carrying a bag shuttle to each classroom of teaching building at many schools, they are in misery that they cannot find a classroom for self-study. Now, with the development of wireless sensor networks, classroom management automation technology is constantly devel- 
oping, and there have many kinds of classroom management systems based images, fingerprints, smart cards and other technology[1]. The main flow of these systems is: classroom demographics, web publishing, students landed inquiry system to obtain information about the current classroom free seats, with a purpose to find the study room. Infrared detection method has also been applied on a number of statistics, and coach overcrowding monitoring system in this article is also based on infrared detection, combined with networking and wireless communications technology, to achieve signal acquisition, display, wireless transmission, and its structure is intact and suitable for many occasions. The monitoring network model is shown in Figure 1

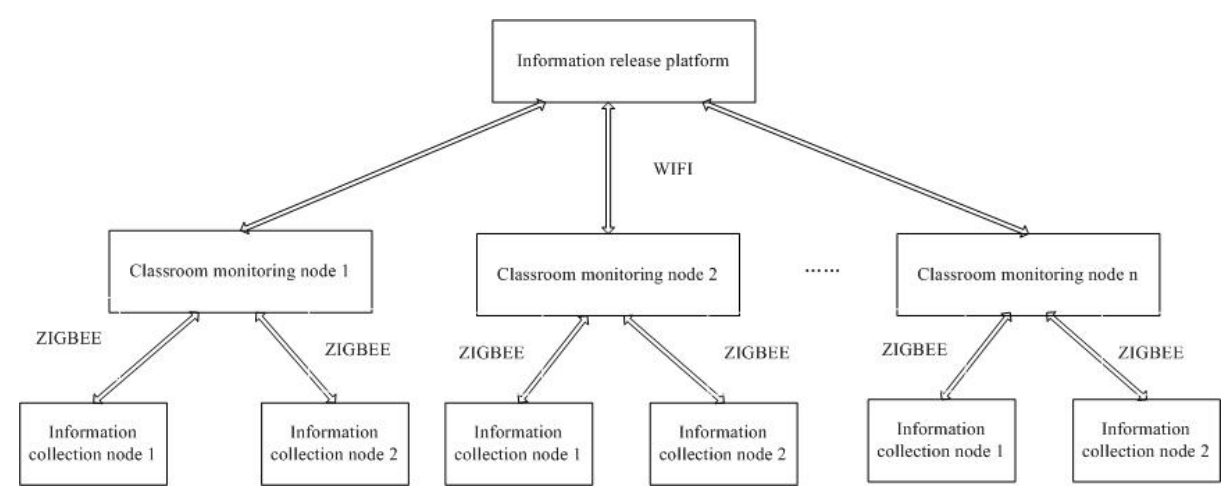

Fig. 1. Block diagram of classroom free seat monitoring system

The system uses pyroelectric infrared sensors to detect whether someone in or out of the classroom, and then the processor control counter plus or minus according to the flow of personnel. Classroom monitoring system consists of three nodes, two collection nodes are located near the two doors of the classroom, the collected information is sent to the aggregation node through the ZIGBEE communication module, and the aggregation node sends the collected information to the information release platform through WIFI. Students can connect WIFI to check which classroom has spare seats after entering the teaching building,.

\section{Design of classroom available seat monitoring system}

The functions of available seat monitoring system is to calculate number of persons and information transmission. As a ZIGBEE system, the task of coordinator is to count the number and start the information transmission part, and the information collection nodes collect the number of in classroom. Currently, there are two doors in a classroom, students who enter the room may be from any one. Therefore, there must be three nodes, which include pyroelectric infrared sensor is placed near the door to judge the students going out or entering the classroom, and the third one collects information with ZIGBEE module which is on the CC2530 chip. In addition, the coordinator can display through LCD. Finally, the coordinator also needs to transmit the 


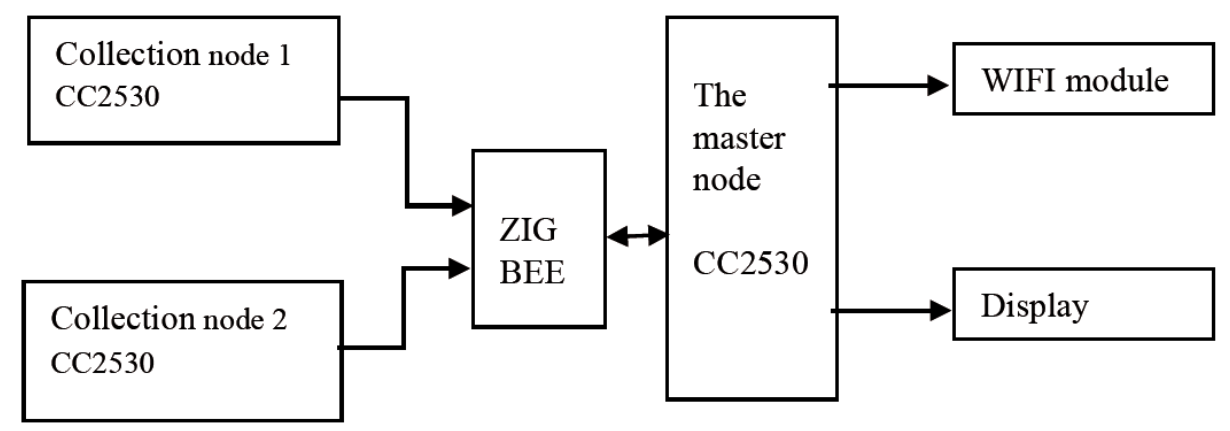

Fig. 2. Block diagram of the classroom automatic selection system

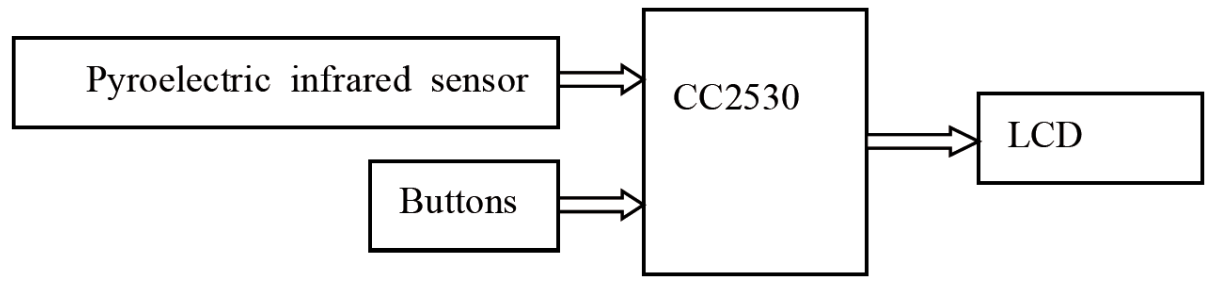

Fig. 3. Block diagram of the subordinate node

collected information to the information release platform through the WIFI module. System design block diagram is shown in Figure 2.

\subsection{Functional design for the subordinate node}

The tasks of the collection node are signal acquisition and transmission. The structure and function block diagram shown in Figure 3.

The signal acquisition device of the collection node is pyroelectric infrared sensor module which includes RE200B and its conditioning circuit ${ }^{[2]}$, and the master chip CC2530 signal responses of the sensor module. It should design a counter to record the number of persons who enter the classroom. When people come around to the sensor, it will appear a signal which is a state different from original state, and the MCU must record the state as a data. Design three buttons are used to control the subordinate node like opening and closing nodes, clear.

\subsection{Pyroelectric effect}

The phenomenon of outside temperature changing lead crystal internally generated spontaneous polarization is termed the pyroelectric effect, and pyroelectric infrared sensor uses the pyroelectric effect to detect infrared radiation in the light-receiving device ${ }^{[3]}$. It is made of ceramic oxides or piezoelectric crystal components, whose two surfaces are made of electrodes. When the temperature changes $\Delta \mathrm{T}$ within the 
sensor monitoring range, the pyroelectric effect will generate charges $\Delta \mathrm{Q}$ on the two electrodes.

$$
\Delta Q=p \Delta T
$$

Where $\mathrm{p}$ is the pyroelectric coefficient, its value is

$$
p=\left(\frac{\partial D}{\partial T}\right)_{E, X}=\frac{-\alpha_{0} D_{0}}{\alpha_{0}\left(T-T_{0}\right)+3 \beta D_{0}^{3}}
$$

Where $\mathrm{D}_{0}$ is the electric displacement field $\mathrm{E}$ at temperature $\mathrm{T}$ caused.

It produces a weak voltage $\Delta \mathrm{V}$ between the two electrodes. Due to its high output impedance, there is a field effect transistor in the sensor impedance transformation. Charge $\Delta \mathrm{Q}$ generated by pyroelectric effect will disappear due to bound the ions in the air, as to say, when the ambient temperature is stable and unchanging, $\Delta \mathrm{T}=0$, the sensor is no output. When the body enters the detection zone, because of the difference temperature between the body and surroundings, it comes an output $\Delta \mathrm{T}$; if the body is not active after entering the detection zone, the temperature does not change, the sensor does not output.

Typical application of pyroelectric infrared signal detection is to install on the sensitive devices. Fresnel lenses are mostly made of polyolefin material which is injection pressure to the sheet, the lens surface is smooth on one side, and the other side burned steeled concentric circles. This Fresnel lens can limit peak frequency of the incident light to about 10 microns (human infrared radiation peak). The roles of the Fresnel lens are the following two: one is focusing effect, pyroelectric infrared signal refraction (reflection) on the sensitive device; the other is divided the detection area into a number of bright and dark areas, that moving object entered the detection area can produce pyroelectric infrared signal on the sensitive devices in the form of a temperature change. It uses special optical lens principle, and engenders an alternating "blind spot" and "sensitive areas" in front of the detector in order to improve its detection and receiver sensitivity. When someone walked from the front of the lens, infrared body would continue to alternate from "blind" into the "sensitive areas", o that the received infrared signal is pulsed input suddenly strong suddenly weak, thereby enhancing its energy margin. Pyroelectric infrared sensor signal acquisition works shown in Figure 4.

Fresnel lens is made of a cap which buckles on the sensitive element when used, and the top cap is engraved with concentric surface. With the Fresnel lens, the sensor module sensing range is shown in Figure 5.

From the above two figures analysis, the sensor can be placed horizontally, can also be placed vertically, and vertically placed cover a larger area. Because the human face and arm infrared radiation is stronger, so that these parts should be just in front of the lens when sensor placed. After calculation and experiment, signal acquisition device should be placed in the door at a height of about 1.5 meters. 

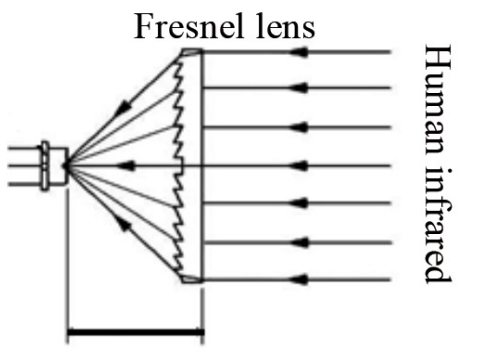

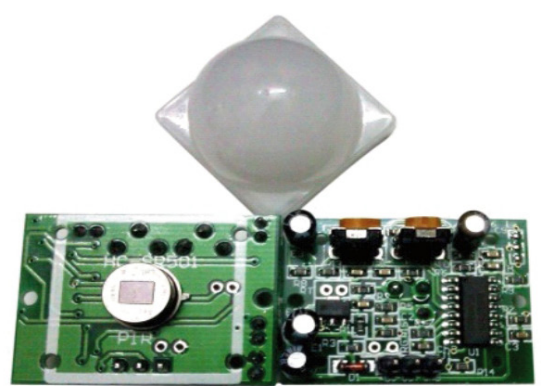

Focal length

Fig. 4. Signal acquisition principle of pyroelectric infrared sensor
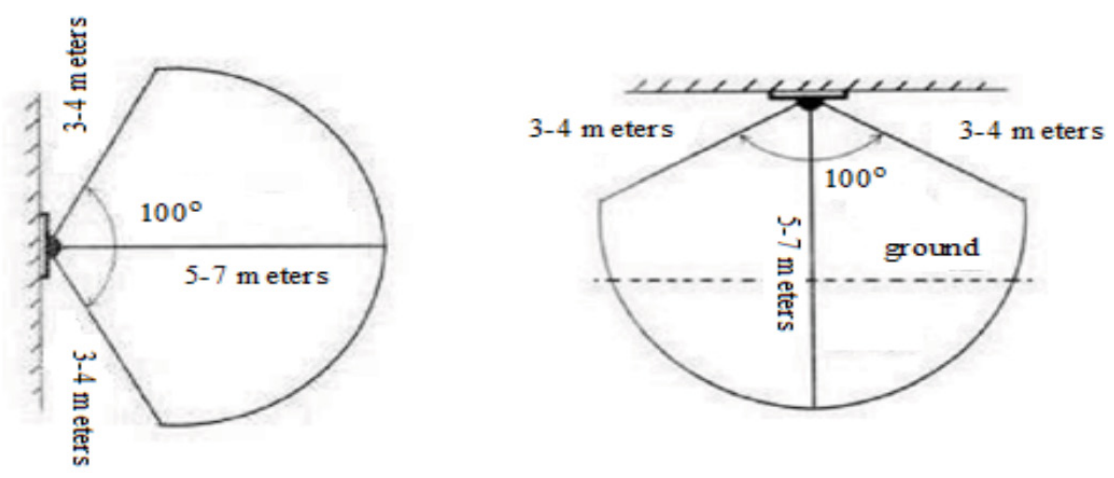

Fig. 5. Sensing range of the sensor module

\subsection{Sensor signal acquisition and processing}

The system uses a dual element pyroelectric infrared sensor RE200B.This sensor uses two opposite polarity sensitive pyroelectric unit fitted on the same wafer, and it not only can play the role of temperature compensation [4], but it can output different characteristics in the direction of body movement waveform. The internal structure of RE200B is shown in Figure 6.

Since the voltage which pyroelectric infrared-sensitive device output is extremely weak and accompanied by numerous noise, it cannot directly be used, and it need for signal preprocessing [5]. This system uses BISS0001 which is a processing chip for processing the sensor signal

After pretreatment the high level of the sensor module output is $3.3 \mathrm{Vand}$ the low level close to $0 \mathrm{~V}$. To enhance the usefulness of the module, it installs delay adjustment potentiometer on the sensor module to control their response time is adjustable between $0.5 \sim 200 \mathrm{~s}$, and installs distance adjustment potentiometer for adjusting signal accuracy. The module's output OUT connects IO port of CC2530. Figure 7a shows the output signal of the sensor which oscilloscope observed when someone through the sensor from $\mathrm{A}$ to $\mathrm{B}$, and figure $7 \mathrm{~b}$ shows the output signal when someone through the sensor from B to A. 


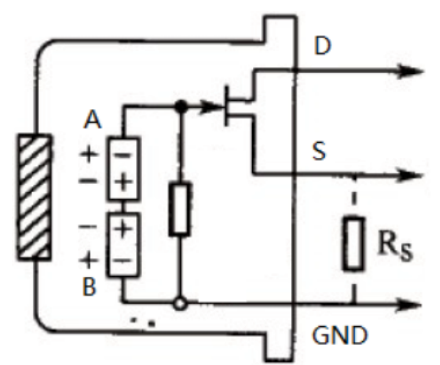

A Forward sensor

B Reverse Sensor

Fig. 6. RE200B pyroelectric infrared

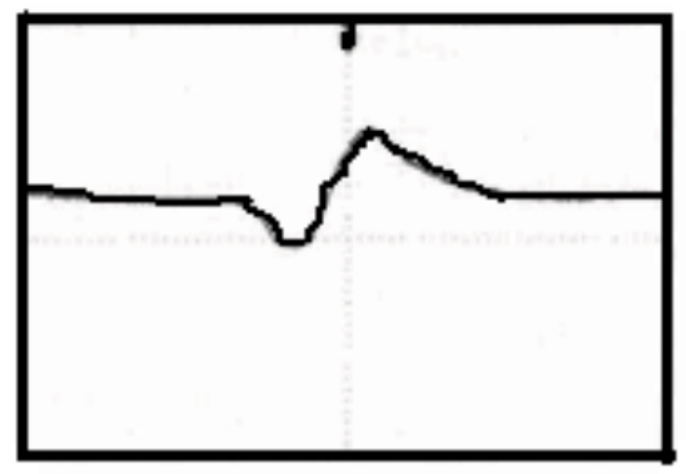

a) Waveform of single direction along $\mathrm{A}$ to $\mathrm{B}$

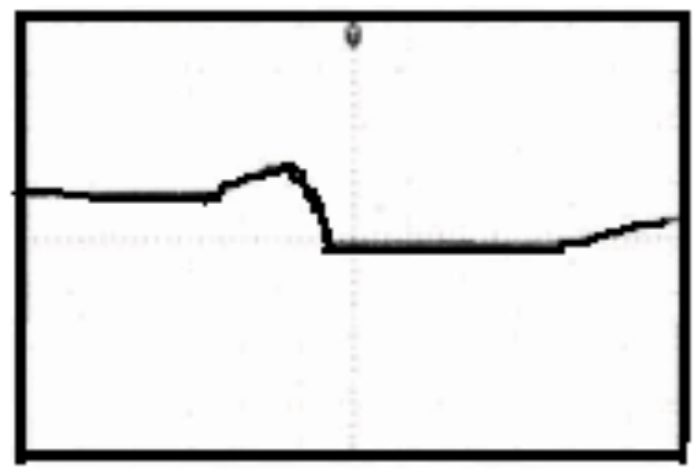

b) Waveform of single direction along B to A

Fig. 7.

The signal acquisition device is sealed and attached to the door of the classroom. When someone enters the room, the sensor's output is falling, the microcontroller call count counting procedures; the other hand, when someone out of the classroom, the counter counts down. 


\subsection{ZIGBEE communication design}

For each classroom information collection system, what should be done is that the coordinator node communicates with multiple acquisition nodes through the RF communication module on the CC2530 chip[6]. Due to the nature of the 802.15.4 protocol, nodes that join the network are automatically assigned network numbers and channels[7]. In order to avoid the adjacent network interconnection error message, the system adds a flag in the communication protocol. When communication is carried out, the connection is established only when the flag of the collection node and the coordinator flag are the same. The procedure of collection node is shown in Figure 8.

If the new network is successful, the coordinator node binds the collection node, allocates and binds the node address, receives data by address, or sends an instruction[8]. At the same time, the coordinator uploads the statistical data to the network terminal. Coordinator received data to be transmitted to the network platform also needs the relay circuit WIFI, so it needs to set the external memory data temporary storage. the Program flow of coordinator of is shown in Figure 9.

\section{WIFI communication design}

The statistics of each classroom's monitoring system are uploaded to the web platform through WIFI. This system uses ESP8266-12WIFI modules to communicate

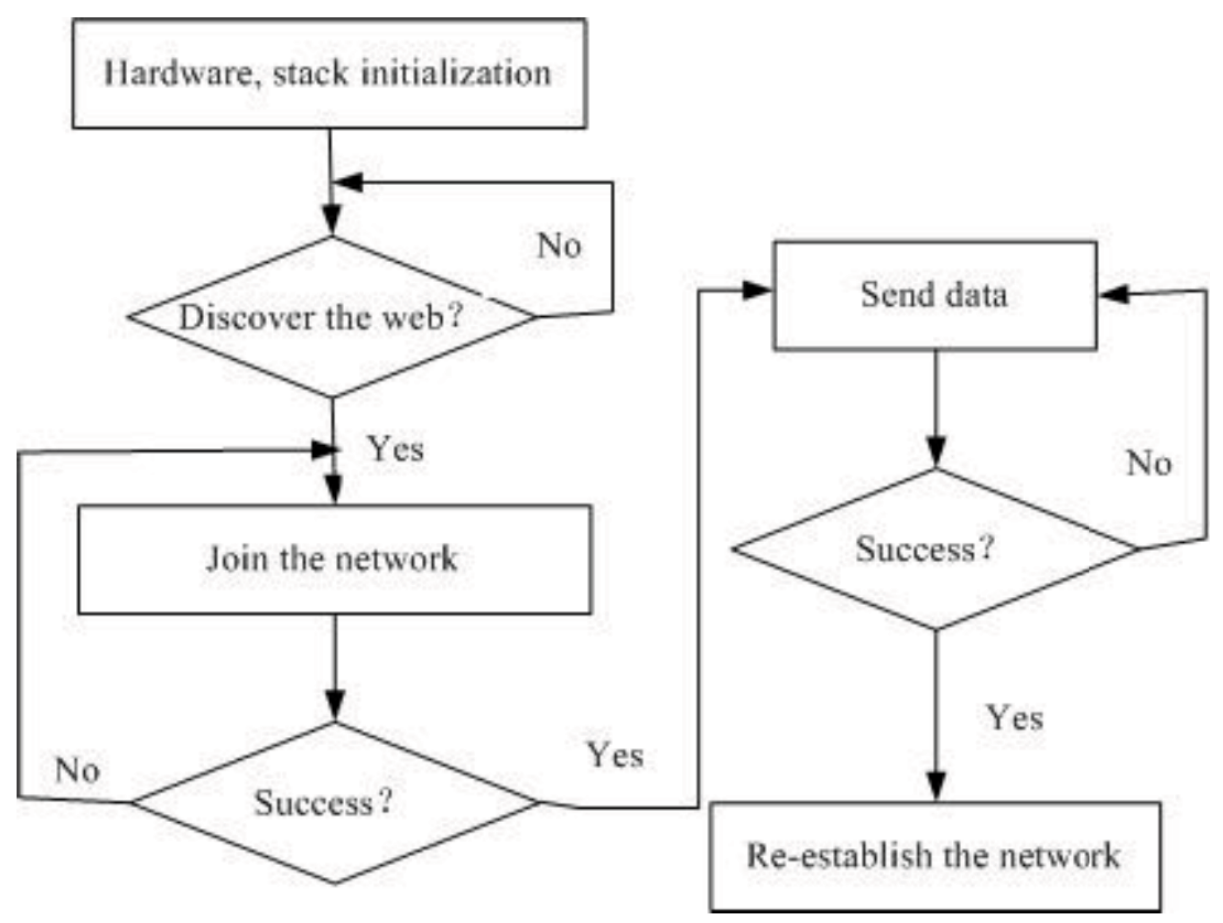

Fig. 8. Collection node flow chart 


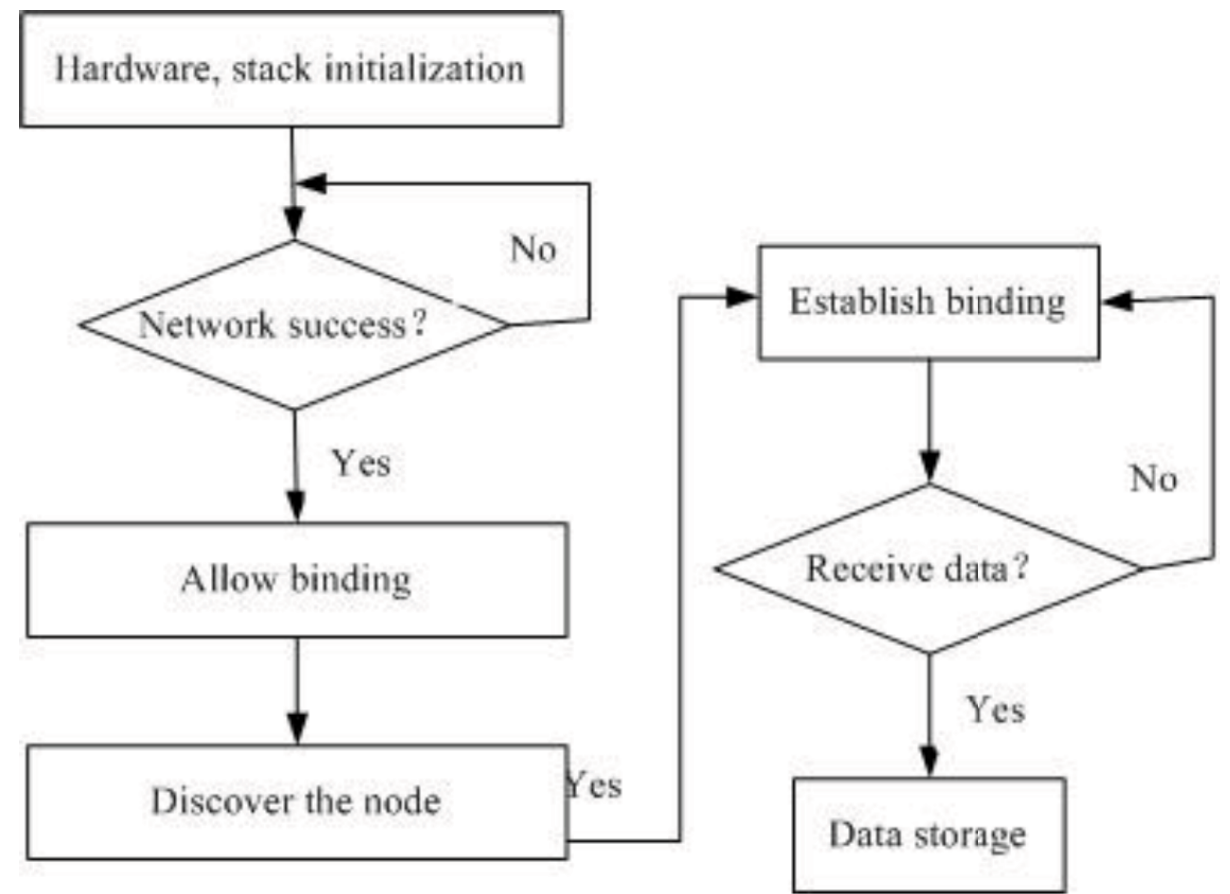

Fig. 9. Coordinator node flow chart

with the microcontroller[9]. The module supports three operating modes as STA / $\mathrm{AP} / \mathrm{STA}+\mathrm{AP}$, supports UART data communication interface, 3.3V single power supply, low power consumption, and it is a highly intelligent wireless communication module. The module has an 8-pin output, except for power and ground connections, the WIFI's RXD connects with the TXD in the microcontroller and WIFI's TXD connects with the RXD in microcontroller, and then it achieves the hardware connection for the microcontroller and the WIFI. In order to achieve the automation of the from node, there is no control key for sending and receiving data, therefore whether or not to send signal is determined by the master node. Meanwhile, this system designs the delay program to control the time of receiving data. When the time that no data transmission over 1s, it considered that the system has stopped data scenting. Process of communication between WIFI modules with the microcontroller is shown in Figure 10 .

Working mode of WIFI can be set by the phone when apply the system in practice. The coordinator nodes are set to STA mode and the network address is set to the slave address. In order to reduce the error rate of data transmission, the crystal frequency of MCU is $11.0592 \mathrm{MHz}$, and serial port baud rate is set to 115200 , the baud rate of WIFI is also needed set to 115200 when debug. 


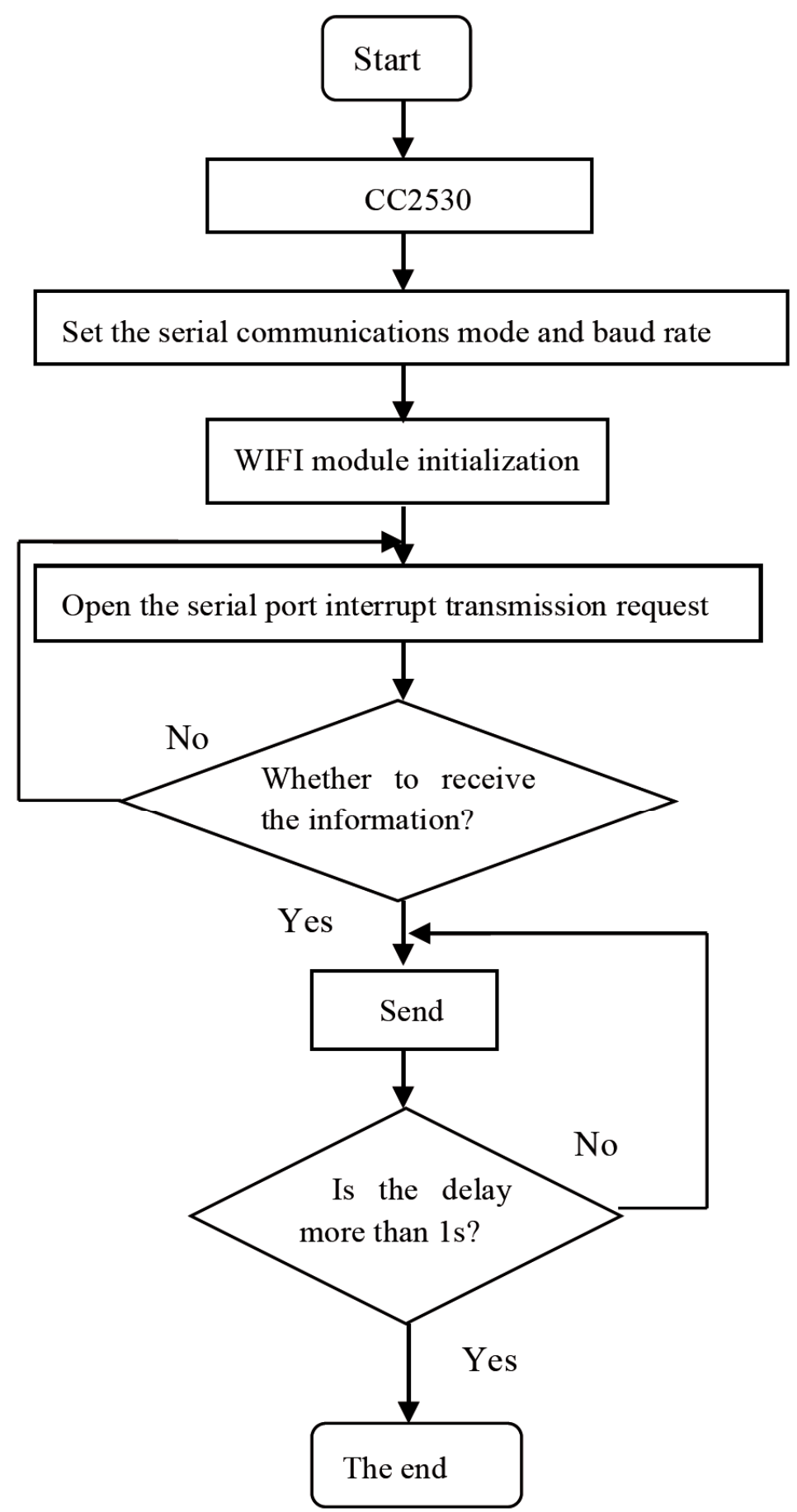

Fig. 10.WIFI transmit data flow 


\section{$4 \quad$ System testing and analysis}

Design test system model is as follows: 10 sensor nodes were installed in 5 classrooms, each classroom installs two nodes in front and back doors, and each classroom set up a coordinator node, the node aggregation router into the corridor, the test data acquisition accuracy and data transfer rate. It tests in the following environments.

The five classrooms are far apart, the signal without interference. The five classrooms is in the same floor, and the plane distance is relatively far. Actual data is the actual number of empty seats, and test data is the number of empty seats tested, and rate is the time that information transfer to the network terminal after the number of empty seats changed. The test data is shown in Table 1.

From the test results in the table, there is data error, and the transmission rate is not the same for each classroom. The reason for the error may be that when two people enter the classroom at the same time, the time interval is too small, mistaken for being a person. In addition, the data transfer rate is related to the distance, and the No. 5 classroom is furthest away from the convergence node.

The five classrooms are adjacent. The five classrooms are on the same floor and adjacent to each other. Do the same test as in the previous step. The test data is shown in Table 2.

As can be seen from the data in that table, the network interference in adjacent classrooms is rather serious. Since each classroom's network works in the $2.4 \mathrm{GHz}$ frequency band, the adjacent classroom network admits of a node. Although the flag bit has been set, the interference is relatively serious with respect to Case (1). However, due to the router node is relatively close to each classroom, the transfer rate has increased.

The five classrooms are on different floors. The five classrooms are on the different floors, but are close by. Do the same test as in the previous step. The test data is shown in Table 3.

As can be seen from the data in that table, when the vertical distance is short, the interference between networks is also serious. And the vertical distance affects the transfer rate.

The five classrooms are scattered on different floors. The five classrooms are on the different floors and are very far away. Do the same test as in the previous step. The test data is shown in Table 4.

As can be seen from the data in the that table, after a long distance, the network interference is almost non-existent, but the transmission time is prolonged.

Two of the five classrooms are in class. The five classrooms are on the different floors and are very far away, and classroom 1 and classroom 2 are in class. Do the same test as in the previous step. The test data is shown in Table 5 .

As can be seen from the data in Table 5, for the classroom which is in class, the data error is too large. Students enter and out of the classroom more concentrated when is in class, and the density is too large, and the sensor identification error is large. Therefore, the drawback of the system is that it can't identify whether the classroom is in class or not. 
Through the analysis of the above test results, it is found that the error in the application of the network construction scheme in empty seats in the classroom is relatively small, and the transmission delay is relatively short, which meets the application requirements.

Table 1.

\begin{tabular}{|l|c|c|c|c|c|}
\hline \multicolumn{1}{|c|}{ Number } & Classroom1 & Classroom2 & Classroom3 & Classroom4 & Classroom5 \\
\hline Actual data & 68 & 79 & 57 & 43 & 32 \\
\hline Test Data & 69 & 78 & 57 & 45 & 32 \\
\hline Rate & $1 \mathrm{~s}$ & $1 \mathrm{~s}$ & $1.2 \mathrm{~s}$ & $1.1 \mathrm{~s}$ & $1.4 \mathrm{~s}$ \\
\hline
\end{tabular}

Table 2.

\begin{tabular}{|l|c|c|c|c|c|}
\hline \multicolumn{1}{|c|}{ Number } & Classroom1 & Classroom2 & Classroom3 & Classroom4 & Classroom5 \\
\hline Actual data & 68 & 79 & 57 & 43 & 32 \\
\hline Test Data & 65 & 73 & 54 & 39 & 28 \\
\hline Rate & $1 \mathrm{~s}$ & $1 \mathrm{~s}$ & $1 \mathrm{~s}$ & $1 \mathrm{~s}$ & $1 \mathrm{~s}$ \\
\hline
\end{tabular}

Table 3.

\begin{tabular}{|l|c|c|c|c|c|}
\hline \multicolumn{1}{|c|}{ Number } & Classroom1 & Classroom2 & Classroom3 & Classroom4 & Classroom5 \\
\hline Actual data & 68 & 79 & 57 & 43 & 32 \\
\hline Test Data & 66 & 76 & 55 & 38 & 29 \\
\hline Rate & $1.1 \mathrm{~s}$ & $1 \mathrm{~s}$ & $1 \mathrm{~s}$ & $1.1 \mathrm{~s}$ & $1 \mathrm{~s}$ \\
\hline
\end{tabular}

Table 4.

\begin{tabular}{|l|c|c|c|c|c|}
\hline \multicolumn{1}{|c|}{ Number } & Classroom1 & Classroom2 & Classroom3 & Classroom4 & Classroom5 \\
\hline Actual data & 68 & 79 & 57 & 43 & 32 \\
\hline Test Data & 68 & 78 & 57 & 41 & 30 \\
\hline Rate & $1.1 \mathrm{~s}$ & $1.3 \mathrm{~s}$ & $1.2 \mathrm{~s}$ & $1.1 \mathrm{~s}$ & $1.2 \mathrm{~s}$ \\
\hline
\end{tabular}

Table 5.

\begin{tabular}{|l|c|c|c|c|c|}
\hline \multicolumn{1}{|c|}{ Number } & Classroom1 & Classroom2 & Classroom3 & Classroom4 & Classroom5 \\
\hline Actual data & 12 & 20 & 57 & 43 & 32 \\
\hline Test Data & 23 & 35 & 57 & 41 & 30 \\
\hline Rate & $1.1 \mathrm{~s}$ & $1.3 \mathrm{~s}$ & $1.2 \mathrm{~s}$ & $1.1 \mathrm{~s}$ & $1.2 \mathrm{~s}$ \\
\hline
\end{tabular}

\section{Conclusions}

This article describes an automatic monitoring system for checking classrooms free seats based on ZIGBEE and WIFI. The network uses a star topology, WIFI and ZIGBEE heterogeneous networking. The collection node detects signals by dual element pyroelectric infrared sensor. It starts different electrode according to people 
walking direction. When students entered the classroom, a falling edge output from the sensor; and rising output when out of the classroom. Therefore, design register of the collection node according to the voltage change of the sensor signal, and calculate the number of students in classroom. And then transmits information to the coordinator by ZIGBEE. The coordinator receives the information and processes them, and then displays them on the LCD. Meanwhile, it sends messages to users upon request information by WIFI.

Through the analysis of the test data, it is found that the error in the application of the network construction scheme in empty seats in the classroom is relatively small, and the transmission delay is relatively short, which meets the application requirements.

The similarities and differences between this system and others currently in use are following:

1. Use the human body detection sensor as a signal acquisition device.

2. Signal transmission indoor is by ZIGBEE. Sensor network identification set distinguish network, in order to enhance anti-interference ability.

3. Transmission of information through WIFI to the users enables a direct service to students who enter the teaching building.

The whole monitoring system automatically recognizes, automatic transmission, to achieve the purpose of the numbers of people in classroom automatic detection and information transformation without human intervention.

\section{Acknowledgment}

1. This work is supported by Hebei Agricultural University Science and Technology Fund(LG201604).

2. This work is supported by Baoding Science Technology Research and Development Guidance Program (15ZG023).

\section{References}

[1] Ling Lin, Gang Li, Ru Ding, "The new microcontroller interface devices and technologies”, Xi'an: Xi'an University of Electronic Science and Technology Press, 2005.

[2] Wei Zhao, "The Researeh of Human Tracking with Pyroeleetric Infrared Sensor Network",. Anhui University, 2012.

[3] Jinqiao Yi, Yong Huang, Honghua Liao, "Pyroelectric infrared sensor and its application in people counting system", Infrared and Laser Engineering, 2015 (4), 1186-1192.

[4] JIN Zhong-chao , SU Ben-yue, JIANG Wei, JIANG Yue "Design and Implementation of Self - Study Room People Number Intelligent Query System Based on Zigbee”, Journal of Guizhou University ( Natural Sciences) 2014 (12), 85-88.

[5] Bo Yang, Youxian Chen, "The principle and applications of Pyroelectric infrared sensors", Instrumentation Technology, 2008 (6), 66-67. 
Paper-Design of Seat Search System in the Classroom Based on Wireless Sensor networks

[6] Moridi, Mohammad, Kawamura, "Development of underground mine monitoring and communication system integrated ZigBee and GIS," International Journal of Mining Science and Technology, 2015 , 25 (5) :811-818 https://doi.org/10.1016/j.ijmst.2015.07.017

[7] Wen jing, Zhang, "The Design of Internet of Things Smart Home System based on S3C2440 and Zigbee", International Journal of Technology Management, $2013: 81-83$.

[8] Guo Xingge, Wang Jiansheng, Liu Zhu. "Design of Libery Study Room Management System Based on High Frequency RFID Technology", Journal of Xuzhou Institute of Technology(Natural Science Edition), 2014 (6), 49-54

[9] Jianming Cao, Fei Hui, "Distributed Monitoring Scene Splicing System Based on WiFi", Computer Systems \& Applications, 2014 (12), 47-52

[10] Yang A.Y, Jafari R, Sastry S.S. "Distributed recognition of human actions using wearable motion sensor networks". Journal of Ambient Intelligence and Smart Environment, 2009, 1( 2) : $103-115$

[11] Z Chen, H Zou, H Jiang. "Fusion of WiFi, Smartphone Sensors and Landmarks Using the Kalman Filter for Indoor Localization". Sensors, 2015,15(1):715-732 https://doi.org/10.3390/s150100715

[12] M Jalilian, H Sariri, F Parandin, MM Karkhanehchi, M Hookari. "Design and implementation of the monitoring and control systems for distribution transformer by using GSM network". International Journal of Electrical Power \& Energy Systems, 2016,74 :36-41 https://doi.org/10.1016/j.ijepes.2015.07.022

[13] A Ibrahim, A Paravath, PK Aswin, SM Iqbal, SU Abdulla. "GSM based digital door lock security system". International Conference on Power , $2016: 1-6$

[14] M Maity, B Raman, M Vutukuru. "TCP Download Performance in Dense WiFi Scenarios: Analysis and Solution". IEEE Transactions on Mobile Computing, 2016,16 (1) :213227 https://doi.org/10.1109/TMC.2016.2540632

[15] G Dandachi, S Elayoubi, T Chahed, N Chendeb. "Network centric versus user centric multihoming strategies in LTE/WiFi networks". IEEE Transactions on Vehicular Technology, 2017,PP (99) :1-1

[16] WU Jian, W Hou, B Zheng. "A Temperature Control System Based on STC89C52 MCU”. Computer Knowledge \& Technology, 2011

\section{Authors}

Yuchen Jia is with the College of Information Science \& Technology, Agricultural University of Hebei, Baoding 071000, China (jiayuchen1981@163.com).

Mengxuan Cui is with the College of life, Beijing Institute of Technology, Beijing, 100081, China.

Article submitted 14 November 2017. Published as resubmitted by the authors 28 December 2017. 